\title{
On the Equivalence of Differential Equations in the Sense of Coincidence Reflecting Functions
}

\author{
Zhengxin Zhou \\ School of Mathematical Sciences, Yangzhou University, Yangzhou 225002, China \\ Correspondence should be addressed to Zhengxin Zhou; zxzhou@yzu.edu.cn \\ Received 7 March 2015; Revised 17 April 2015; Accepted 20 April 2015 \\ Academic Editor: Jaume Giné
}

Copyright (C) 2015 Zhengxin Zhou. This is an open access article distributed under the Creative Commons Attribution License, which permits unrestricted use, distribution, and reproduction in any medium, provided the original work is properly cited.

We use a new method for constructing some differential equations which are equivalent to a given equation in the sense of having the same reflecting function. We completely solve the problem: when is a polynomial differential equation equivalent to a given polynomial differential equation? Many sufficient conditions have been established for one differential equation to be equivalent to a given differential equation. We apply the obtained results to study the boundary value problem of two equivalent differential equations.

\section{Introduction}

By $[1,2]$ we know that some polynomial differential system

$$
\begin{aligned}
& \dot{u}=U(u, v), \\
& \dot{v}=V(u, v),
\end{aligned}
$$

where $U(u, v)$ and $V(u, v)$ are polynomial functions, can be transformed to an equation of the form

$$
\begin{array}{r}
\frac{d x}{d t}=p_{0}(t)+p_{1}(t) x+\cdots+p_{n}(t) x^{n}=P(t, x) \\
t, x \in \mathbb{R},
\end{array}
$$

where $p_{i}(t)$ are polynomial in $\cos t, \sin t$. The fact that systems with a homogeneous nonlinearity can be transformed to (2) with $n=3$ has been exploited in a number of previous papers [1-4]. The limit cycles of (1) correspond to $2 \pi$-periodic solutions of (2). This fact has been used to facilitate certain computations and to provide some information about the global phase portrait of the system. In this paper we will use the method of Mironenko [5] to construct some differential equations which are equivalent to (2) in the sense of having the same reflecting function.

Now, we simply introduce the concept of the reflecting function.
Consider differential system

$$
x^{\prime}=X(t, x), \quad t \in \mathbb{R}, x \in D \subset \mathbb{R}^{n},
$$

which has a continuously differentiable right-hand side and general solution $\varphi\left(t ; t_{0}, x_{0}\right)$. For each such system, the reflecting function is defined as $F(t, x):=\varphi(-t, t, x)[5]$.

If system (3) is $2 \pi$-periodic with respect to $t$, then $T(x):=$ $F(-\pi, x)$ is the Poincaré mapping of (3) over the period $[-\pi, \pi]$. Thus, the solution $x=\varphi\left(t ;-\pi, x_{0}\right)$ of (3) defined on $[-\pi, \pi]$ is $2 \pi$-periodic if and only if $x_{0}$ is a fixed point of $T(x)$.

A differentiable function $F(t, x)$ is a reflecting function of system (3) if and only if it is a solution of the Cauchy problem

$$
\begin{array}{r}
F_{t}(t, x)+F_{x}(t, x) X(t, x)+X(-t, F(t, x))=0, \\
F(0, x)=x .
\end{array}
$$

If the reflecting functions of two differential systems coincide in their common domain, then these systems are said to be equivalent [5].

If $F(t, x)$ is the reflecting function of (3), then it is also the reflecting function of any system

$$
x^{\prime}=X(t, x)+F_{x}^{-1} R(t, x)-R(-t, F(t, x)),
$$

where $R(t, x)$ is an arbitrary vector function such that the solutions of every system above are uniquely determined by 
its initial conditions. The systems (3) and (5) have the same operators of translation along solutions on the symmetric time interval $[-\omega, \omega]$, and therefore the initial data $x(-\omega)$ of solutions of the boundary value problems of the form $\Phi(x(\omega), x(-\omega))=0$, where $\Phi$ is an arbitrary function, coincide for such systems.

In general, it is very difficult to find out the reflecting function of (3), so to writ out system (5) is difficult, too. How to judge if two systems are equivalent when we do not know their reflecting function? This is a very interesting problem! Mironenko in [5-7] has studied it and obtained some good results.

Lemma 1 (see [6]). If continuously differentiable vector functions $\Delta_{i}(t, x)(i=1,2, \ldots, m)$ are solutions of differential system

$$
\Delta_{t}(t, x)+\Delta_{x}(t, x) X(t, x)=X_{x}(t, x) \Delta(t, x),
$$

then all perturbed systems of the form

$$
x^{\prime}=X(t, x)+\sum_{i=1}^{m} \alpha_{i}(t) \Delta_{i}(t, x),
$$

where $\alpha_{i}(t)$ are arbitrary continuous scalar odd functions, are equivalent to each other and to system (3).

The theory of reflecting function has been used for studying the qualitative behavior of solutions of differential systems by many authors [5-13].

Now, for a given equation (2), we present a method for constructing other first-order differential equations which are equivalent to (2), that is, to find out the solution of (6) with $X(t, x)=P(t, x)$. However, it is impossible to find all the solutions in most cases. Therefore, we take only polynomial solutions of (6), that is, its solutions of the form

$$
\Delta=a_{0}(t)+a_{1}(t) x+\cdots+a_{n}(t) x^{n},
$$

where the coefficients $a_{i}(t)(i=0,1,2, \ldots, n)$ are assumed to be differentiable functions on $\mathbb{R}$ and $a_{n}(t)$ is not to be zero identically.

Bel'skii in papers [8-10] has discussed, respectively, when a first-order polynomial differential equation is equivalent to a linear equation $(n=1)$ and Riccati equation $(n=2)$ and Abel equation $(n=3)$. In this paper, we will in detail discuss when is a first-order equation equivalent to (2) with $n \geq 3$. We have obtained the sufficient conditions for (6) has solution in form of (8) and we give the explicit expressions of $\Delta(t, x)$. At the same time, in addition, we also find out some nonpolynomial solutions of (6). The results of this paper generalize the results of Bel'skii [8-10], and the method of proof in this paper is more simple and efficient than in the previous papers.

\section{Main Results}

Now, we consider (2) and assume that $p_{n}(t)$ is not to be zero identically in $\mathbb{R}$ and $n>1$. By Bel'skii [8], we know that if $p_{n} \neq 0$, then the degree of polynomial $\Delta(t, x)$ is equal to $n$. In the following, we suppose that $n \geq 3$ and $p_{n}(t) \neq 0$.
Theorem 2. For (2), suppose that $n \geq 3$ and $p_{n}(t) \neq 0$. Then the function $\Delta(t, x)$ of the form (8) is a solution of (6), if and only if

$$
\begin{aligned}
a_{0}^{\prime}= & p_{1} a_{0}-a_{1} p_{0}, \\
a_{k}^{\prime}= & (k+1)\left(p_{k+1} a_{0}-a_{k+1} p_{0}\right) \\
& +(k-1)\left(p_{k} a_{1}-p_{1} a_{k}\right), \quad k=1,2, \ldots, n-2, \\
a_{i}= & \frac{p_{i}}{p_{n}} a_{n}, \quad i=2,3, \ldots, n-1, \\
a_{1}= & \frac{p_{1}}{p_{n}} a_{n}+\frac{a_{n}^{\prime}}{(n-1) p_{n}}, \\
a_{0}= & \frac{p_{0}}{p_{n}} a_{n}+\frac{a_{n}}{n p_{n}}\left(\frac{p_{n-1}}{p_{n}}\right)^{\prime}+\frac{a_{n}^{\prime} p_{n-1}}{n(n-1) p_{n}^{2}} .
\end{aligned}
$$

Therefore, $\Delta$ can be represented in the form

$$
\begin{aligned}
\Delta= & \frac{a_{n}}{p_{n}} P(t, x)+\frac{a_{n}^{\prime}}{(n-1) p_{n}}\left(x+\frac{p_{n-1}}{n p_{n}}\right) \\
& +\frac{a_{n}}{n p_{n}}\left(\frac{p_{n-1}}{p_{n}}\right)^{\prime} .
\end{aligned}
$$

Proof. Let us substitute the function $\Delta(t, x)$ of the form (8) into (6); we get

$$
\begin{aligned}
a_{0}^{\prime} & +a_{1}^{\prime} x+\cdots+a_{n}^{\prime} x^{n}+\left(a_{1}+2 a_{2} x+\cdots+n a_{n} x^{n-1}\right) \\
& \cdot\left(p_{0}+p_{1} x+\cdots+p_{n} x^{n}\right) \\
& =\left(p_{1}+2 p_{2} x+\cdots+n p_{n} x^{n-1}\right) \\
& \cdot\left(a_{0}+a_{1} x+\cdots+a_{n} x^{n}\right) .
\end{aligned}
$$

Equating the coefficients of the like power of $x$, we obtain

$$
a_{k}^{\prime}=\sum_{i=1}^{k+1} i\left(p_{i} a_{k+1-i}-a_{i} p_{k+1-i}\right),
$$

$$
k=0,1,2, \ldots, 2 n-1,
$$

where $a_{k}=0$ and $p_{k}=0$, when $k>n$.

From (16) when $k=2 n-1$ we get $n a_{n} p_{n}=n p_{n} a_{n}$. Taking $k=2 n-2$ in (16), we have $a_{n-1}=\left(p_{n-1} / p_{n}\right) a_{n}$. Similarly for $k=2 n-3,2 n-4, \ldots, n+1$, we get

$$
a_{i}=\frac{p_{i}}{p_{n}} a_{n}, \quad i=2,3, \ldots, n-1
$$

that is, relation (11) is true.

Substituting (11) into (16) and simplifying we get (10).

Using relation (11) and taking $k=n$ in (16), we get

$$
a_{n}^{\prime}=(n-1)\left(a_{1} p_{n}-p_{1} a_{n}\right) ;
$$

it implies relation (12). 
Using relation (12) and taking $k=n-1$ in (16), we have

$$
a_{n-1}^{\prime}=n\left(p_{n} a_{0}-p_{0} a_{n}\right)+\frac{n-2}{n-1} \frac{p_{n-1}}{p_{n}} a_{n}^{\prime}
$$

substituting $a_{n-1}=\left(p_{n-1} / p_{n}\right) a_{n}$ into the above, we get that relation (13) is held.

Substituting (11)-(13) into (8), we get

$$
\begin{aligned}
\Delta(t, x)= & \frac{a_{n}}{p_{n}}\left(p_{0}+p_{1} x+\cdots+p_{n} x^{n}\right) \\
& +\frac{a_{n}^{\prime}}{(n-1) p_{n}}\left(x+\frac{p_{n-1}}{n p_{n}}\right)+\frac{a_{n}}{n p_{n}}\left(\frac{p_{n-1}}{p_{n}}\right)^{\prime} \\
= & \frac{a_{n}}{p_{n}} P(t, x)+\frac{a_{n}^{\prime}}{(n-1) p_{n}}\left(x+\frac{p_{n-1}}{n p_{n}}\right) \\
& +\frac{a_{n}}{n p_{n}}\left(\frac{p_{n-1}}{p_{n}}\right)^{\prime},
\end{aligned}
$$

which completes the proof.

We rewrite $P(t, x)$ as follows:

$$
\begin{aligned}
& P(t, x)=p_{n}\left(\left(x+\frac{p_{n-1}}{n p_{n}}\right)^{n}+\delta_{n-2}\left(x+\frac{p_{n-1}}{n p_{n}}\right)^{n-2}\right. \\
& \left.+\cdots+\delta_{1}\left(x+\frac{p_{n-1}}{n p_{n}}\right)+\delta_{0}\right),
\end{aligned}
$$

in which

$$
\begin{aligned}
& \delta_{k}=\left.\frac{1}{k ! p_{n}} \frac{\partial^{k} P(t, x)}{\partial x^{k}}\right|_{\substack{x=-p_{n-1} / n p_{n}\\
}}, \\
& \quad k=0,1, \ldots, n-2, \delta_{n-1} \equiv 0 .
\end{aligned}
$$

Lemma 3. If $\delta_{i}=0, i=k+1, k+2, \ldots, n-2, k \geq 0$, then

$$
\begin{aligned}
\delta_{k} & =\frac{p_{k}}{p_{n}}-C_{n}^{k}\left(\frac{p_{n-1}}{n p_{n}}\right)^{n-k}, \\
\frac{p_{k+1}}{p_{n}} & =C_{n}^{k+1}\left(\frac{p_{n-1}}{n p_{n}}\right)^{n-k-1} .
\end{aligned}
$$

Proof. As $\delta_{i}=0, i=k+1, k+2, \ldots, n-1$, so (21) goes to

$$
\begin{aligned}
\frac{P(t, x)}{p_{n}}= & \left(x+\frac{p_{n-1}}{n p_{n}}\right)^{n}+\delta_{k}\left(x+\frac{p_{n-1}}{n p_{n}}\right)^{k} \\
& +\delta_{k-1}\left(x+\frac{p_{n-1}}{n p_{n}}\right)^{k-1}+\cdots+\delta_{0}
\end{aligned}
$$

equating the coefficients of $x^{k}$ and $x^{k+1}$; from here we get relations (23) and (24).
Theorem 4. Suppose that $\delta_{i}=0, i=k+1, k+2, \ldots, n-2$, $\delta_{k} \neq 0(1<k<n-1)$, and

$$
\begin{aligned}
& (m+1) p_{m+1} \mu_{k 0}+(m-1) p_{m} \mu_{k 1} \\
& =\left(\frac{p_{m}}{p_{n}}\right)^{\prime}-\frac{n-1}{n-k} \frac{p_{m}}{p_{n}} \frac{\delta_{k}^{\prime}}{\delta_{k}}, \quad(m=2,3, \ldots, k-1), \\
& a_{0}^{\prime}=p_{1} a_{0}-a_{1} p_{0}, \\
& a_{1}^{\prime}=2 p_{2} a_{0}-2 p_{0} a_{2}, \\
& a_{m}=\frac{p_{m}}{p_{n}} a_{n}, \quad(m=2,3, \ldots, n-1),
\end{aligned}
$$

where

$$
\begin{aligned}
\mu_{k 0} & =\frac{1}{n p_{n}}\left(\frac{p_{n-1}}{p_{n}}\right)^{\prime}-\frac{p_{n-1}}{n(n-k) p_{n}^{2}} \frac{\delta_{k}^{\prime}}{\delta_{k}} \\
\mu_{k 1} & =-\frac{\delta_{k}^{\prime}}{(n-k) p_{n} \delta_{k}}, \\
a_{0} & =\frac{p_{0}}{p_{n}} a_{n}+\mu_{k 0} a_{n}, \\
a_{1} & =\frac{p_{1}}{p_{n}} a_{n}+\mu_{k 1} a_{n}, \\
a_{n} & =\delta_{k}^{-(n-1) /(n-k)} .
\end{aligned}
$$

Then the function $\Delta(t, x)$ in (8) is a solution of (6); that is, (2) is equivalent to equation

$$
x^{\prime}=P(t, x)+\alpha(t) \Delta(t, x),
$$

where $\alpha(t)$ is an arbitrary continuous odd function.

Proof. For $k \leq m \leq n-2$, by Lemma 3, substituting (12) and (13) into (10), we obtain

$$
\begin{aligned}
a_{m}^{\prime} & \\
= & (m+1)\left(p_{m+1} a_{0}-a_{m+1} p_{0}\right) \\
& +(m-1)\left(p_{m} a_{1}-a_{m} p_{1}\right) \\
= & (m+1)\left(\frac{p_{m+1}}{n p_{n}}\left(\frac{p_{n-1}}{p_{n}}\right)^{\prime} a_{n}+\frac{p_{m+1}}{n(n-1)} \frac{p_{n-1}}{p_{n}^{2}} a_{n}^{\prime}\right) \\
& +(m-1) \frac{p_{m} a_{n}^{\prime}}{(n-1) p_{n}} .
\end{aligned}
$$

Substituting (24) into this relation, we obtain

$$
\begin{aligned}
a_{m}^{\prime}= & \frac{m+1}{n-m} C_{n}^{m+1}\left(\left(\frac{p_{n-1}}{n p_{n}}\right)^{n-m}\right)^{\prime} a_{n} \\
& +\frac{m+1}{n-1} C_{n}^{m+1}\left(\frac{p_{n-1}}{n p_{n}}\right)^{n-m} a_{n}^{\prime}+\frac{m-1}{n-1} \frac{p_{m}}{p_{n}} a_{n}^{\prime} .
\end{aligned}
$$


By (11) and (23), we get

$$
a_{n} \delta_{m}^{\prime}+\frac{n-m}{n-1} \delta_{m} a_{n}^{\prime}=0 .
$$

As $\delta_{m}=0(m=k+1, k+2, \ldots, n-1),(37)$ is an identity when $m=k+1, k+2, \ldots, n-1$. For $m=k$, solving (37), we have

$$
a_{n}=\delta_{k}^{-(n-1) /(n-k)} .
$$

Substituting (38) into (11)-(13), we obtain (29)-(32).

On the other hand, by relation (10), for $1<m<k$, we have

$$
\begin{aligned}
a_{m}^{\prime}= & (m+1)\left(p_{m+1} a_{0}-a_{m+1} p_{0}\right) \\
& +(m-1)\left(p_{m} a_{1}-a_{m} p_{1}\right) .
\end{aligned}
$$

Substituting (29)-(32) and (37) into the above, we obtain

$$
\begin{aligned}
& \left(\frac{p_{m}}{p_{n}}\right)^{\prime} a_{n}-\frac{n-1}{n-k} \frac{p_{m} a_{n} \delta_{k}^{\prime}}{p_{n} \delta_{k}} \\
& =(m+1) p_{m+1} \mu_{k 0} a_{n}+(m-1) p_{m} \mu_{k 1} a_{n},
\end{aligned}
$$

which deduces that relation (26) is true. By Theorem 2 and in (10) taking $k=1$ we get that relations (27) and (28) are held.

By Lemma 1 and Theorem 2, the proof is completed.

Theorem 5. Supposing that $n>3$,

$$
\begin{aligned}
& \delta_{i}=0, \quad i=2,3, \ldots, n-2, \\
& \delta_{1}=\frac{p_{1}}{p_{n}}-C_{n}^{1}\left(\frac{p_{n-1}}{n p_{n}}\right)^{n-1} \neq 0, \\
& \eta^{\prime} a_{n}+\frac{n}{n-1} \eta a_{n}^{\prime}=0, \\
& a_{k}=\frac{p_{k}}{p_{n}} a_{n}, \quad(k=2,3, \ldots, n-1) \\
& a_{0}=\left(\frac{p_{0}}{p_{n}}-\frac{p_{n-1} \delta_{1}}{n p_{n}}+\frac{1}{n p_{n}}\left(\frac{p_{n-1}}{p_{n}}\right)^{\prime}\right) a_{n}+\frac{\lambda_{0} p_{n-1}}{n p_{n}}, \\
& a_{1}=n\left(\frac{p_{n-1}}{n p_{n}}\right)^{n-1} a_{n}+\lambda_{0},
\end{aligned}
$$

where

$$
\begin{aligned}
\eta: & \frac{p_{0}}{p_{n}}-\left(\frac{p_{n-1}}{n p_{n}}\right)^{n}+\frac{1}{p_{n}}\left(\frac{p_{n-1}}{n p_{n}}\right)^{\prime}-\frac{p_{n-1}}{n p_{n}} \delta_{1}, \\
a_{n} & =e^{-(n-1) \int \delta_{1} p_{n} d t}\left[\lambda_{1}\right. \\
& \left.+\lambda_{0}(n-1) \int p_{n} e^{(n-1) \int \delta_{1} p_{n} d t} d t\right],
\end{aligned}
$$

where $\lambda_{0}$ and $\lambda_{1}$ are arbitrary constants. Then the function $\Delta(t, x)$ of the form (8) is a solution of (6); that is, (2) is equivalent to (34).
Proof. As $\delta_{m}=0, m=2,3, \ldots, n-1$ and $\delta_{1} \neq 0$, by Lemma 3, we have

$$
\begin{aligned}
& \delta_{1}=\frac{p_{1}}{p_{n}}-C_{n}^{1}\left(\frac{p_{n-1}}{n p_{n}}\right)^{n-1}, \\
& \frac{p_{2}}{p_{n}}=C_{n}^{2}\left(\frac{p_{n-1}}{n p_{n}}\right)^{n-2} .
\end{aligned}
$$

Using $\delta_{m}=0(m=2,3, \ldots, n-1)$, it is not difficult to check that (37) is an identity when $m=2,3, \ldots, n-2$. For $m=1$, in relation (10) taking $k=1$ and computing, it follows that

$$
a_{1}^{\prime}=n\left(a_{n}\left(\frac{p_{n-1}}{n p_{n}}\right)^{n-1}\right)^{\prime},
$$

which implies that

$$
a_{1}=n\left(\frac{p_{n-1}}{n p_{n}}\right)^{n-1} a_{n}+\lambda_{0} .
$$

Combining relation (12), it yields

$$
a_{n}^{\prime}=-(n-1) \delta_{1} p_{n} a_{n}+(n-1) \lambda_{0} p_{n}
$$

solving this equation we get the expression (45).

Substituting (49) into (13) implies (42). Substituting (42)(45) into equation $a_{0}^{\prime}=p_{1} a_{0}-p_{0} a_{1}$ and simplifying we get $\eta^{\prime} a_{n}+(n /(n-1)) \eta a_{n}^{\prime}=0$. By Theorem 2 , the proof is finished.

Similarly, we get the following.

Theorem 6. Supposing that $\delta_{m}=0, m=1,2, \ldots, n-2$ and $\delta_{0}=p_{0} / p_{n}-\left(p_{n-1} / n p_{n}\right)^{n} \neq 0$,

$$
\begin{aligned}
& \left(\delta_{0}+\frac{1}{n p_{n}}\left(\frac{p_{n-1}}{p_{n}}\right)^{\prime}\right)^{\prime} a_{n} \\
& +\frac{n}{n-1} a_{n}^{\prime}\left(\delta_{0}+\frac{1}{n p_{n}}\left(\frac{p_{n-1}}{p_{n}}\right)^{\prime}\right)=0, \\
& a_{k}=\frac{p_{k}}{p_{n}} a_{n}, \quad k=2,3, \ldots, n-1, \\
& a_{0}=\frac{p_{0}}{p_{n}} a_{n}+\frac{a_{n}}{n p_{n}}\left(\frac{p_{n-1}}{p_{n}}\right)^{\prime}+\frac{p_{n-1}}{n p_{n}} \lambda_{0}, \\
& a_{1}=\frac{p_{1}}{p_{n}} a_{n}+\lambda_{0},
\end{aligned}
$$

where

$$
a_{n}=(n-1) \lambda_{0} \int p_{n} d t+\lambda_{1}
$$

$\lambda_{0}$ and $\lambda_{1}$ are arbitrary constants. Then (2) is equivalent to (34). 
Theorem 7. Supposing that $\delta_{m}=0, m=0,1,2, \ldots, n-2$,

$$
\begin{aligned}
a_{n}\left(\frac{p_{n-1}}{p_{n}}\right)^{\prime}+\lambda_{0} p_{n-1} & =\lambda_{2} n p_{n}, \\
a_{0} & =\frac{p_{0}}{p_{n}} a_{n}+\lambda_{2}, \\
a_{1} & =\frac{p_{1}}{p_{n}} a_{n}+\lambda_{0}, \\
a_{k} & =\frac{p_{k}}{p_{n}} a_{n}, \quad k=2,3, \ldots, n-1,
\end{aligned}
$$

where

$$
a_{n}=(n-1) \lambda_{0} \int p_{n} d t+\lambda_{1}
$$

$\lambda_{i}(i=0,1,2)$ are arbitrary constants. Then (2) is equivalent to (34).

By the literature [5], we know the following.

Corollary 8. If all the conditions of one of the above four theorems (Theorems 4-7) are satisfied and (2) is $2 \pi$-periodic in $t$, then the initial data $x(-\pi)$ of solutions of the boundary value problems of the form $\Phi(x(\pi), x(-\pi))=0$, where $\Phi$ is an arbitrary function, coincide for systems (2) and (34).

Example 9. The equation

$$
x^{\prime}=(x-\sin t)^{4}+\cos t
$$

has the only one $2 \pi$-periodic solution $x=\sin t$. For this equation, we have

$$
\begin{aligned}
& p_{0}=\cos t+\sin ^{4} t, \\
& p_{1}=-4 \sin ^{3} t, \\
& p_{2}=6 \sin ^{2} t, \\
& p_{3}=-4 \sin t, \\
& p_{4}=1, \\
& \delta_{3}=\delta_{2}=\delta_{1}=0, \\
& \delta_{0}=\cos t .
\end{aligned}
$$

By Theorem 6, we get

$$
a_{4}=3 \lambda_{0} t+\lambda_{1}
$$

where $\lambda_{0}$ and $\lambda_{1}$ are constants:

$$
\begin{aligned}
& a_{3}=-4 a_{4} \sin t, \\
& a_{2}=6 a_{4} \sin ^{2} t, \\
& a_{1}=-4 a_{4} \sin ^{3} t+\lambda_{0}, \\
& a_{0}=a_{4} \sin ^{4} t-\lambda_{0} \sin t .
\end{aligned}
$$

As

$$
\delta_{0}+\frac{1}{4 p_{4}}\left(\frac{p_{3}}{p_{4}}\right)^{\prime}=\cos t-\cos t=0,
$$

then

$$
\begin{aligned}
& \left(\delta_{0}+\frac{1}{4 p_{4}}\left(\frac{p_{3}}{p_{4}}\right)^{\prime}\right)^{\prime} a_{4}+\frac{4}{3} a_{4}^{\prime}\left(\delta_{0}+\frac{1}{4 p_{4}}\left(\frac{p_{3}}{p_{4}}\right)^{\prime}\right) \\
& =0
\end{aligned}
$$

that is, the first condition of Theorem 6 is satisfied. Thus

$$
\Delta_{0}=\left(3 \lambda_{0} t+\lambda_{1}\right)(x-\sin t)^{4}+\lambda_{0}(x-\sin t) .
$$

Taking $\lambda_{0}=0$ and $\lambda_{1}=1$, we get

$$
\Delta_{1}=(x-\sin t)^{4} \text {. }
$$

Taking $\lambda_{0}=1$ and $\lambda_{1}=0$, we have

$$
\Delta_{2}=3 t(x-\sin t)^{4}+(x-\sin t) .
$$

Therefore, (54) is equivalent to equation

$$
\begin{aligned}
x^{\prime}= & (x-\sin t)^{4}+\cos t+\alpha_{0}(t) \Delta_{0}+\alpha_{1}(t) \Delta_{1} \\
& +\alpha_{2}(t) \Delta_{2},
\end{aligned}
$$

where $\alpha_{i}(t)(i=0,1,2)$ are arbitrary continuously odd functions. By Corollary 8, (63) has only one solution such that $x(-\pi)=x(\pi)$.

Now, taking $n=3$ in the above theorems, we get the following corollaries.

Corollary 10. Suppose that $p_{3} \neq 0$; then

$$
\begin{aligned}
\zeta^{\prime} a_{3}+\frac{3}{2} \zeta a_{3}^{\prime}= & 0, \\
\zeta:= & \frac{p_{0}}{p_{3}}-\left(\frac{p_{2}}{3 p_{3}}\right)^{3}+\frac{1}{p_{3}}\left(\frac{p_{2}}{3 p_{3}}\right)^{\prime} \\
& -\frac{p_{2}}{3 p_{3}} \sigma_{1}, \\
a_{0}= & \left(\frac{p_{0}}{p_{3}}-\frac{p_{2}}{3 p_{3}} \sigma_{1}\right) a_{3}+\frac{a_{3}}{3 p_{3}}\left(\frac{p_{2}}{p_{3}}\right)^{\prime} \\
& +\frac{p_{2}}{3 p_{3}} \lambda_{0}, \\
a_{1}= & \frac{p_{2}^{2}}{3 p_{3}^{2}} a_{3}+\lambda_{0}, \\
a_{2}= & \frac{p_{2}}{p_{3}} a_{3},
\end{aligned}
$$

where

$$
a_{3}=e^{-2 \int \sigma_{1} p_{3} d t}\left[\lambda_{1}+2 \lambda_{0} \int p_{3} e^{2 \int a_{3} \sigma_{1} d t} d t\right]
$$


$\lambda_{0}$ and $\lambda_{1}$ are arbitrary constants; $\sigma_{1}=p_{1} / p_{3}-p_{2}^{2} / 3 p_{3}^{2}$. Then the Abel equation

$$
x^{\prime}=p_{0}+p_{1} x+p_{2} x^{2}+p_{3} x^{3}
$$

is equivalent to equation

$$
\begin{aligned}
x^{\prime}= & p_{0}+p_{1} x+p_{2} x^{2}+p_{3} x^{3} \\
& +\alpha(t)\left(a_{0}+a_{1} x+a_{2} x^{2}+a_{3} x^{3}\right),
\end{aligned}
$$

where $\alpha(t)$ is an arbitrary continuously odd function.

Corollary 11. Suppose that $p_{3} \neq 0, \sigma_{1}=p_{1} / p_{3}-p_{2}^{2} / 3 p_{3}^{2}=0$, and $\sigma_{0}=p_{0} / p_{3}-p_{2}^{3} / 27 p_{3}^{3} \neq 0$;

$$
\begin{aligned}
& \left(\sigma_{0}+\frac{1}{3 p_{3}}\left(\frac{p_{2}}{p_{3}}\right)^{\prime}\right)^{\prime} a_{3}+\frac{3}{2} a_{3}^{\prime}\left(\sigma_{0}+\frac{1}{3 p_{3}}\left(\frac{p_{2}}{p_{3}}\right)^{\prime}\right) \\
& =0 \\
& a_{0}=\frac{p_{0}}{p_{3}} a_{3}+\frac{a_{3}}{3 p_{3}}\left(\frac{p_{2}}{p_{3}}\right)^{\prime}+\frac{p_{2}}{3 p_{3}} \lambda_{0}, \\
& a_{1}=\frac{p_{1}}{p_{3}} a_{3}+\lambda_{0}, \\
& a_{2}=\frac{p_{2}}{p_{3}} a_{3},
\end{aligned}
$$

where

$$
a_{3}=2 \lambda_{0} \int p_{3} d t+\lambda_{1}
$$

$\lambda_{0}$ and $\lambda_{1}$ are arbitrary constants. Then Abel equation (66) is equivalent to (67).

Corollary 12. Suppose that $p_{3} \neq 0, \sigma_{1}=p_{1} / p_{3}-p_{2}^{2} / 3 p_{3}^{2}=0$, and $\sigma_{0}=p_{0} / p_{3}-p_{2}^{3} / 27 p_{3}^{3}=0$;

$$
\begin{aligned}
a_{3}\left(\frac{p_{2}}{p_{3}}\right)^{\prime}+\lambda_{0} p_{2} & =3 \lambda_{2} p_{3}, \\
a_{0} & =\frac{p_{0}}{p_{3}} a_{3}+\lambda_{2}, \\
a_{1} & =\frac{p_{1}}{p_{3}} a_{3}+\lambda_{0}, \\
a_{2} & =\frac{p_{2}}{p_{3}} a_{3},
\end{aligned}
$$

where

$$
a_{3}=2 \lambda_{0} \int p_{3} d t+\lambda_{1}
$$

$\lambda_{i}(i=0,1,2)$ are arbitrary constants. Then Abel equation (66) is equivalent to (67).

Example 13. Abel equation

$$
x^{\prime}=x^{3}+6 x^{2}+3 x-10=(x+2)^{3}-9(x+2)
$$

has three constant solutions $x_{1}(t)=-2, x_{2}(t)=-5$, and $x_{3}(t)=1$. For this equation, we have $p_{0}=-10, p_{1}=3, p_{2}=6$, $p_{3}=1$, and $\sigma_{1}=-9$.

Thus

$$
\begin{aligned}
\zeta & =\frac{p_{0}}{p_{3}}-\left(\frac{p_{2}}{3 p_{3}}\right)^{3}+\frac{1}{p_{3}}\left(\frac{p_{2}}{3 p_{3}}\right)^{\prime}-\frac{p_{2}}{3 p_{3}} \sigma_{1} \\
& =-18+18=0
\end{aligned}
$$

and $\zeta^{\prime} a_{3}+(3 / 2) \zeta a_{3}^{\prime}=0$.

Solving $a_{3}^{\prime}=18 a_{3}+2 \lambda_{0}$, we get

$$
a_{3}=\lambda_{1} e^{18 t}-\frac{1}{9} \lambda_{0}
$$

where $\lambda_{0}, \lambda_{1}$ are arbitrary constants. Therefore

$$
\begin{aligned}
a_{1}= & 3\left(\lambda_{1} e^{18 t}-\frac{1}{9} \lambda_{0}\right)+9 \lambda_{1} e^{18 t}, \\
a_{0}= & -10\left(\lambda_{1} e^{18 t}-\frac{1}{9} \lambda_{0}\right)+18 \lambda_{1} e^{18 t}, \\
\Delta_{0}= & \left(\lambda_{1} e^{18 t}-\frac{1}{9} \lambda_{0}\right)\left(x^{3}+6 x^{2}+3 x-10\right) \\
& +9 \lambda_{1} e^{18 t}(x+2) .
\end{aligned}
$$

Taking $\lambda_{1}=0$ and $\lambda_{1}=-9$, we obtain

$$
\Delta_{1}=x^{3}+6 x^{2}+3 x-10
$$

Taking $\lambda_{0}=0$ and $\lambda_{1}=1$, we get

$$
\Delta_{2}=e^{18 t}\left(x^{3}+6 x^{2}+12 x+8\right) \text {. }
$$

Consequently, by Lemma 1 and Corollary 10, Abel equation (72) is equivalent to equation

$$
\begin{aligned}
x^{\prime}= & x^{3}+6 x^{2}+3 x-10+\alpha_{0}(t) \Delta_{0}+\alpha_{1}(t) \Delta_{1} \\
& +\alpha_{2}(t) \Delta_{2},
\end{aligned}
$$

where $\alpha_{i}(t)(i=0,1,2)$ are arbitrary continuously odd functions. By Corollary 8, (78) has only three solutions such that $x(-a)=x(a)$ ( $a$ is a nonzero constant).

Remark 14. The conclusions of the above three corollaries have been proven by Bel'skii and Mironenko in [9].

Because (6) is a linear equation, if $\Delta_{i}(i=1,2, \ldots, m)$ are solutions of (6), then $\Delta=\sum_{i=1}^{m} C_{i} \Delta_{i}$ also is solution of (6), where $C_{i}(i=1,2, \ldots, m)$ are arbitrary constants. In the following, we will find out some solutions $\Delta_{i}$ of (6) in the other form.

Theorem 15. For the first-order differential equation $x^{\prime}=$ $X(t, x)(t, x \in \mathbb{R})$, if $\Delta_{i}(i=1,2, \ldots, m)$ are solutions of $(6)$, then $\Delta=\Delta^{k_{1}} \Delta^{k_{2}} \cdots \Delta^{k_{m}}, \sum_{i=1}^{m} k_{i}=1$, is a solution of (6), too. 
Proof. As

$$
\frac{\partial \Delta_{i}}{\partial t}+\frac{\partial \Delta_{i}}{\partial x} X(t, x)-\frac{\partial X}{\partial x} \Delta_{i}=0, \quad(i=1,2, \ldots, m)
$$

and $k_{1}+k_{2}+\cdots+k_{m}=1$,

$$
\begin{aligned}
& \frac{\partial \Delta}{\partial t}+\frac{\partial \Delta}{\partial x} X(t, x)-\frac{\partial X}{\partial x} \Delta=k_{1} \Delta_{1}^{k_{1}-1} \Delta_{2}^{k_{2}} \\
& \quad \cdots \Delta_{m}^{k_{m}}\left(\frac{\partial \Delta_{1}}{\partial t}+\frac{\partial \Delta_{1}}{\partial x} X(t, x)-\frac{\partial X}{\partial x} \Delta_{1}\right) \\
& \quad+k_{2} \Delta_{1}^{k_{1}} \Delta_{2}^{k_{2}-1} \\
& \quad \cdots \Delta_{m}^{k_{m}}\left(\frac{\partial \Delta_{2}}{\partial t}+\frac{\partial \Delta_{2}}{\partial x} X(t, x)-\frac{\partial X}{\partial x} \Delta_{2}\right)+\cdots \\
& \quad+k_{m} \Delta_{1}^{k_{1}} \Delta_{2}^{k_{2}} \\
& \quad \cdots \Delta_{m}^{k_{m}-1}\left(\frac{\partial \Delta_{m}}{\partial t}+\frac{\partial \Delta_{m}}{\partial x} X(t, x)-\frac{\partial X}{\partial x} \Delta_{m}\right)=0 .
\end{aligned}
$$

Thus, the proof is completed.

By Example 9 and Theorem 15 we get that equation

$$
x^{\prime}=(x-\sin t)^{4}+\cos t
$$

is also equivalent to equation

$$
x^{\prime}=(x-\sin t)^{4}+\cos t+\sum_{i=1}^{m} \alpha_{i}(t) \Delta_{0}^{k_{i 0}} \Delta_{1}^{k_{i 1}} \Delta_{2}^{1-k_{i 0}-k_{i 1}},
$$

where $\alpha_{i}(t)(i=1,2, \ldots, m)$ are arbitrary continuously odd functions, $k_{i 0}, k_{i 1}$ are arbitrary constants, and $\Delta_{i}(i=0,1,2)$ are the same as in Example 9. This equation has only one solution such that $x(-\pi)=x(\pi)$.

Using Theorem 15 and Corollary 10 we get the following.

Corollary 16. Supposing that $p_{3} \neq 0$,

$$
\zeta:=\frac{p_{0}}{p_{3}}-\left(\frac{p_{2}}{3 p_{3}}\right)^{3}+\frac{1}{p_{3}}\left(\frac{p_{2}}{3 p_{3}}\right)^{\prime}-\frac{p_{2}}{3 p_{3}} \sigma_{1}=0 .
$$

Then Abel equation

$$
x^{\prime}=p_{0}+p_{1} x+p_{2} x^{2}+p_{3} x^{3}
$$

is equivalent to equation

$$
\begin{aligned}
x^{\prime}= & p_{0}+p_{1} x+p_{2} x^{2}+p_{3} x^{3}+\alpha_{1} \Delta+\alpha_{2} \Delta_{1}+\alpha_{3} \Delta_{2} \\
& +\alpha_{4} \Delta_{1}^{k} \Delta_{2}^{1-k},
\end{aligned}
$$

where $\alpha_{i}(t)(i=1,2,3,4)$ are arbitrary continuously odd functions and $k$ is a constant:

$$
\Delta=a_{0}+a_{1} x+a_{2} x^{2}+a_{3} x^{3}
$$

in which

$$
\begin{aligned}
& a_{0}=\left(\frac{p_{0}}{p_{3}}-\frac{p_{2}}{3 p_{3}} \sigma_{1}\right) a_{3}+\frac{a_{3}}{3 p_{3}}\left(\frac{p_{2}}{p_{3}}\right)^{\prime}+\frac{p_{2}}{3 p_{3}} \lambda_{0}, \\
& a_{1}=\frac{p_{2}^{2}}{3 p_{3}^{2}} a_{3}+\lambda_{0}, \\
& a_{2}=\frac{p_{2}}{p_{3}} a_{3}, \\
& a_{3}=e^{-2 \int \sigma_{1} p_{3} d t}\left[\lambda_{1}+2 \lambda_{0} \int p_{3} e^{2 \int a_{3} \sigma_{1} d t} d t\right] ;
\end{aligned}
$$

$\lambda_{0}, \lambda_{1}$ are arbitrary constants, and $\sigma_{1}=p_{1} / p_{3}-p_{2}^{2} / 3 p_{3}^{2}$. Consider

$$
\begin{aligned}
& \Delta_{i}=a_{0 i}+a_{1 i} x+a_{2 i} x^{2}+a_{3 i} x^{3}, \quad(i=1,2) \\
& a_{j 1}=\left.a_{j}\right|_{\lambda_{0}=1, \lambda_{1}=0}, \\
& a_{j 2}=\left.a_{j}\right|_{\lambda_{0}=0, \lambda_{1}=1},
\end{aligned}
$$

$$
(j=0,1,2,3) \text {. }
$$

Theorem 17. For equation

$$
\begin{gathered}
x^{\prime}=\beta(t) f(x), \quad t, x \in \mathbb{R}, f(x) \neq 0, \\
\Delta=f(x) \phi\left(\int \frac{1}{f(x)} d x-\int \beta(t) d t\right)
\end{gathered}
$$

is a solution of (6). Thus, (89) is equivalent to equation

$$
x^{\prime}=\beta(t) f(x)+\alpha(t) \Delta(t, x),
$$

where $\alpha(t)$ is an arbitrary continuously odd function, $\phi(u)$ is an arbitrary differentiable function, and $\beta(t)$ is a continuous function.

This result is easy to be proven.

Obviously, from Theorem 17, we see that if $f(x)$ is a polynomial of $x$, then the corresponding equation (6) has at least one polynomial solution $\Delta=f(x)$. This is implied by taking $\phi(u)=1$ in $(90)$.

By Theorem 17, (72) is also equivalent to

$$
\begin{aligned}
x^{\prime}= & \left(x^{3}+6 x^{2}+3 x-10\right) \\
& \cdot\left(1+\alpha(t) \phi\left(\frac{(x+2)^{2}-9}{(x+2)^{2}} e^{-18 t}\right)\right) .
\end{aligned}
$$

If in (92) we put $\phi(u)=1 /\left(1+u^{2}\right)$, then we obtain

$$
\begin{aligned}
x^{\prime} & =\left(x^{3}+6 x^{2}+3 x-10\right) \\
& \left(1+\alpha(t) \frac{(x+2)^{4} e^{36 t}}{(x+2)^{4} e^{36 t}+\left((x+2)^{2}-9\right)^{2}}\right),
\end{aligned}
$$

where $\alpha(t)$ is an arbitrary continuously odd function. This equation has only three solutions such that $x(-a)=x(a)(a$ is a nonzero constant). 


\section{Conflict of Interests}

The author declares that there is no conflict of interests regarding the publication of this paper.

\section{Acknowledgments}

The work was supported by the NSF of Jiangsu of China under Grant no. BK2012682 and the NSF of China under Grant nos. 11271026 and 61374010.

\section{References}

[1] J. Devlin, N. G. Lloyd, and J. M. Pearson, "Cubic systems and Abel equations," Journal of Differential Equations, vol. 147, no. 2, pp. 435-454, 1998.

[2] L. A. Cherkas, "On the number of limit cycles of an autonomous second-order system," Differential Equations, vol. 12, pp. 944946, 1976.

[3] A. L. Neto, "On the number of solutions of the equations $d x / d t=\sum_{j=0}^{n} a_{j}(t) x^{j}, 0 \leq t \leq 1$; for which $x(0)=x(1), "$ Inventiones Mathematicae, vol. 59, pp. 67-76, 1980.

[4] Y. Lijun and T. Yuan, "Some new results on Abel equations," Journal of Mathematical Analysis and Applications, vol. 261, no. 1, pp. 100-112, 2001.

[5] V. I. Mironenko, Analysis of Reflective Function and Multivariate Differential System, University Press, Gomel, Belarus, 2004.

[6] V. V. Mironenko, "Time-symmetry-preserving perturbations of differential systems," Differential Equations, vol. 40, no. 10, pp. 1395-1403, 2004.

[7] V. I. Mironenko and V. V. Mironenko, "Time symmetries and in-period transformations," Applied Mathematics Letters, vol. 24, no. 10, pp. 1721-1723, 2011.

[8] V. A. Bel'skii, "On the construction of first-order polynomial differential equations equivalent to a given equation in the sense of having the same reflective function," Differential Equations, vol. 48, no. 1, pp. 13-20, 2012.

[9] V. A. Bel'skii and V. I. Mironenko, "Reflecting function preserving polynomial perturbations of Abel equation," Problems of Physics, Mathematics and Technics, vol. 9, no. 4, pp. 79-85, 2011.

[10] V. A. Bel'skii and V. I. Mironenko, "Constructing of Abel equation equivalent to the equation of the form $x^{\prime}=A(t)\left(\xi_{0}+\right.$ $\left.\xi_{1} x+\xi_{2} x^{2}+\xi_{3} x^{3}\right)$," Problems of Physics, Mathematics and Technics, vol. 11, no. 2, pp. 55-61, 2012.

[11] E. V. Musafirov, "Differential systems, the mapping over period for which is represented by a product of three exponential matrixes," Journal of Mathematical Analysis and Applications, vol. 329, no. 1, pp. 647-654, 2007.

[12] S. V. Maiorovskaya, "Quadratic systems with a linear reflecting function," Differential Equations, vol. 45, no. 2, pp. 271-273, 2009.

[13] Z.X. Zhou, "On the Poincaré mapping and periodic solutions of nonautonomous differential systems," Communications on Pure and Applied Analysis, vol. 6, no. 2, pp. 541-547, 2007. 


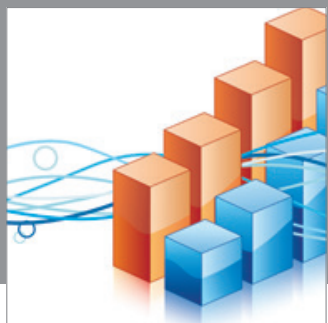

Advances in

Operations Research

mansans

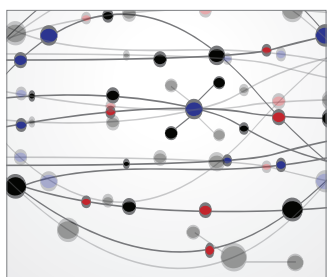

The Scientific World Journal
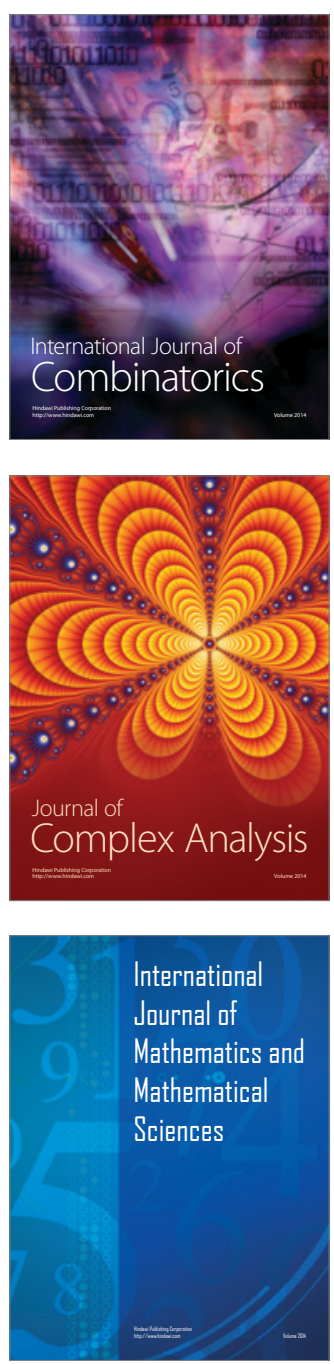
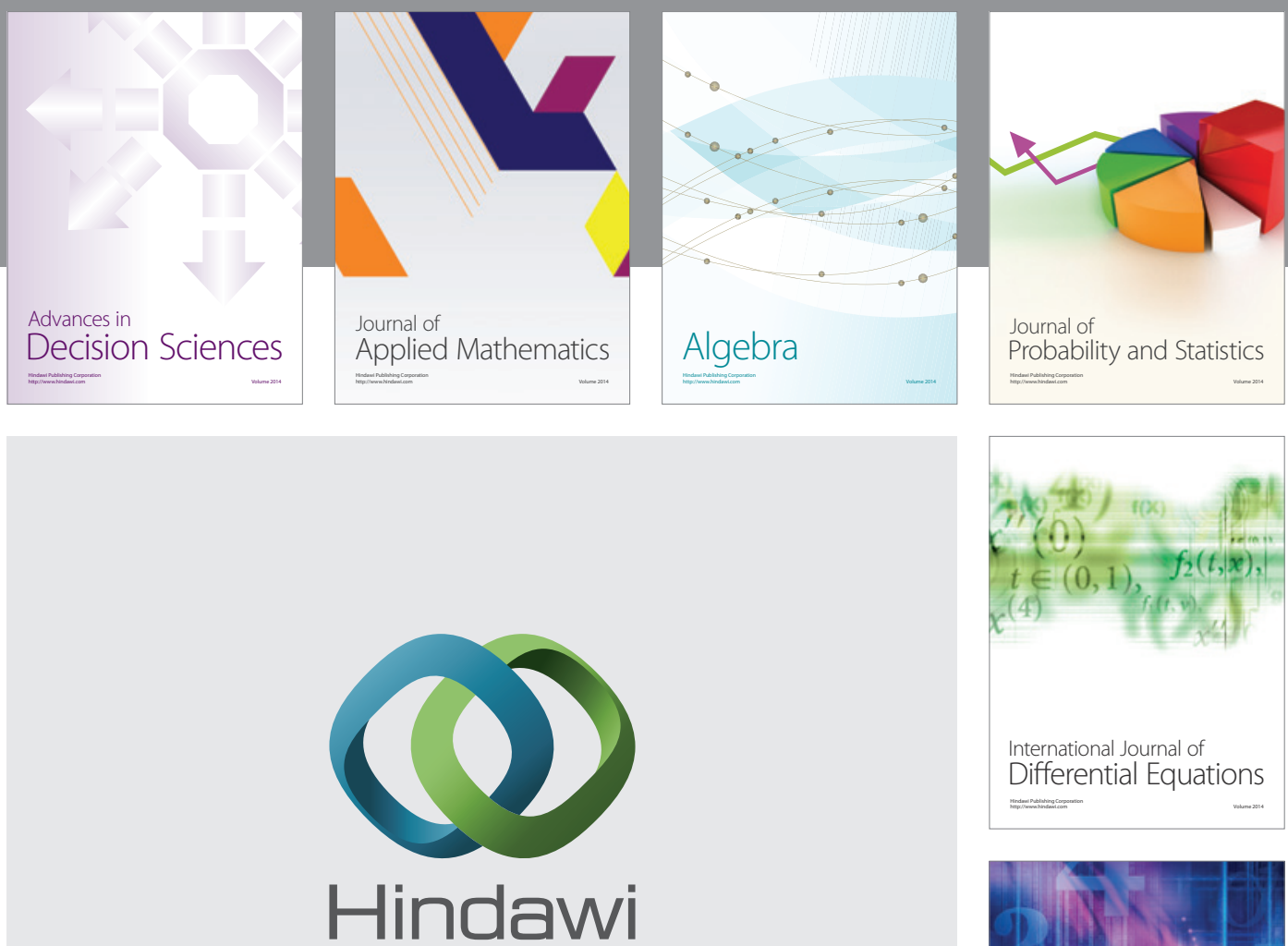

Submit your manuscripts at http://www.hindawi.com
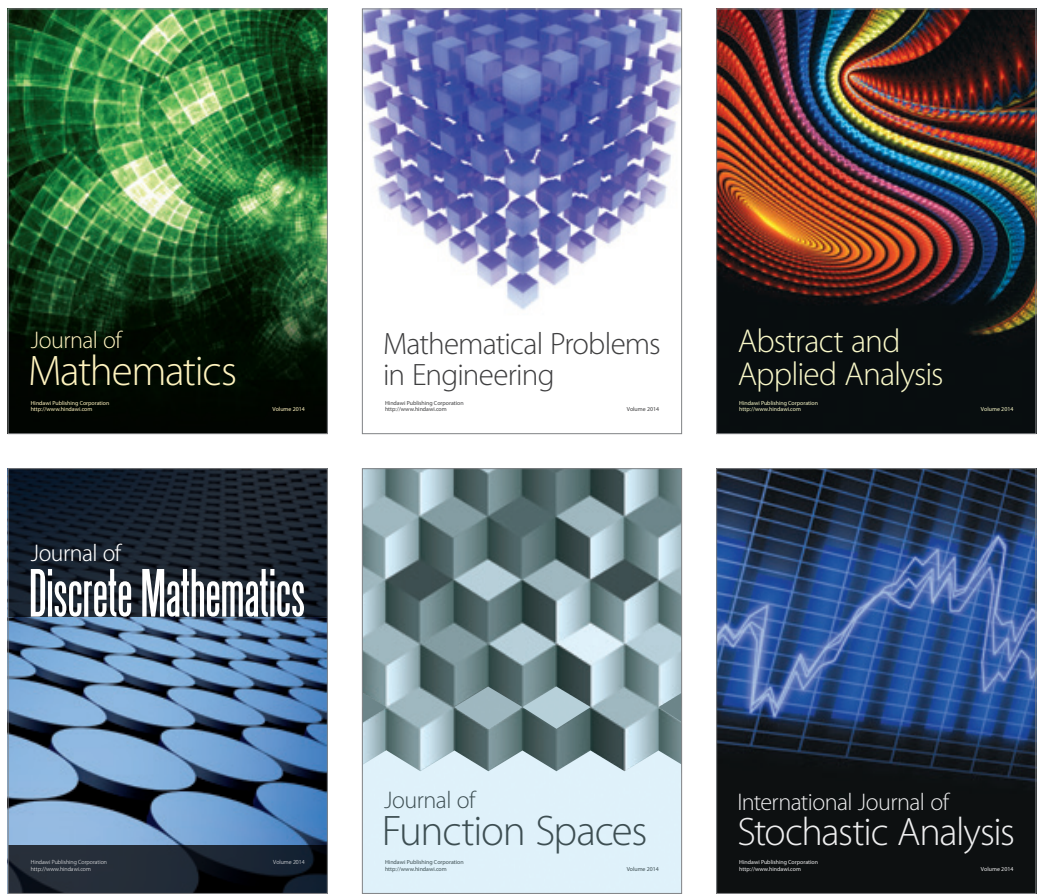

Journal of

Function Spaces

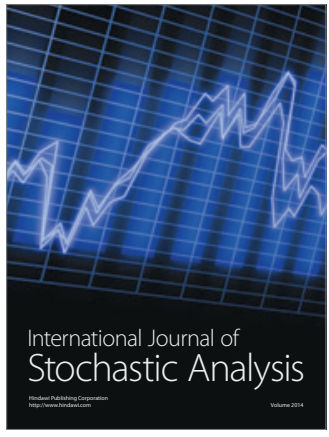

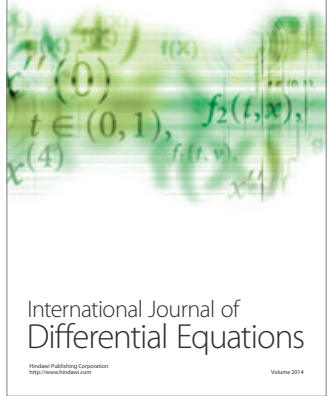
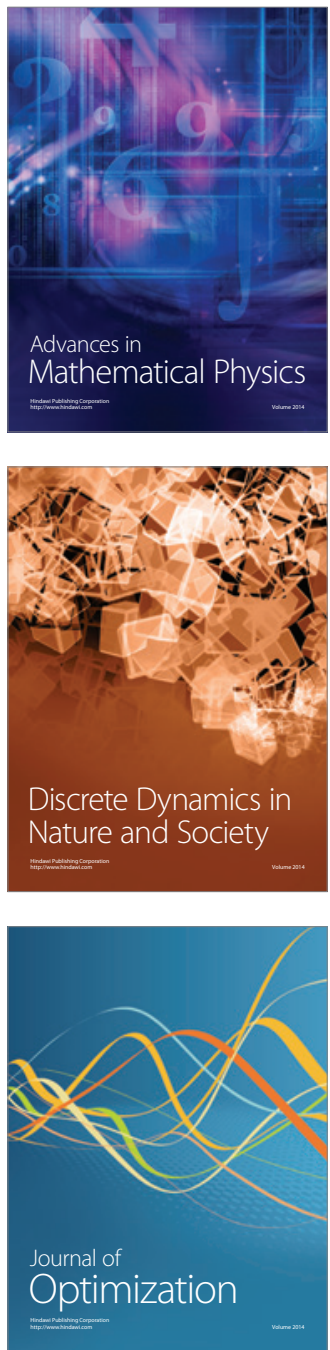